\title{
Compte-rendu
}

\section{Bayart, Jean-François. Les Études postcoloniales, un carnaval académique. Paris : Karthala, 2010.}

\author{
$\mathrm{C}(\mathrm{h})$ ris Reyns-Chikuma \\ Université de l'Alberta
}

Jean-François Bayart est un éminent politologue et un africaniste de renommée internationale. Il est l'auteur et le co-auteur de nombreux articles publiés dans des revues prestigieuses et de plusieurs ouvrages influents tel L'Etat en Afrique: la politique du ventre (1989, suivie de deux rééditions en 1993 et 2006). Certains de ces ouvrages ont été traduits dans plusieurs langues dont l'anglais tel Le Gouvernement du monde: une critique politique de la globalisation (2004) paru en 2007 sous le titre Global Subjects: A Political Critique of Globalization.

Le dernier livre, de Jean-François Bayart, Les Etudes postcoloniales, un carnaval académique, est indéniablement À LIRE pour toutes les personnes intéressées par les «Postcolonial Studies». Sa bibliographie est vaste (99-124). Sa connaissance du sujet est indéniable et nombreuses de ses critiques sont intéressantes. Certaines même sont nouvelles et me semblent tout à fait justifiées. Il est impossible de parler de tous ces aspects positifs ici ou même de les énumérer. Mais je commencerai mon compte-rendu en reprenant quelquesuns de ses aspects positifs pour en montrer aussi les faiblesses. Ainsi, par exemple Bayart, après d'autres, rappelle que l'institution de l'esclavage arabe n'est jamais citée à côté de celle de l'esclavage "atlantique» dans les textes des Postcolonial Studies (11). Cependant, pour être valables ces comparaisons ou bien doivent être systématiques et demanderaient alors des études spécifiques (et il y en a) ou bien elles se font juste en quelques lignes pour rappeler que l'esclavage n'est pas seulement une institution "blanche", mais elles sont alors douteuses délaissant les différences quantitatives et/ou qualitatives. Elles permettent alors des dérapages plus que problématiques comme l'affaire Olivier Pétré-Grenouilleau l'a montré (cf., Smouts 393). Il en va de même du fait important justement rappelé par Bayart que «la violence et l'exclusion dont a été porteuse la République se retrouvent en métropole » (63). Mais dans un tel énoncé, où est le sens des proportions: pour des centaines de morts dus à la répression en métropole, combien de dizaines milliers de morts et d'amputés et de violées dans l'Empire, voire de centaines de milliers ou de millions si l'on compte la traite négrière en Afrique?

En dépit de nombreux arguments intéressants, on ne peut s'empêcher de penser que ce court essai [95p], à la fois polémique et savant, s’inscrit dans un mouvement qui en France refuse de considérer les Postcolonial Studies comme un domaine qui, malgré ses faiblesses et même ses erreurs, a fortement contribué à renouveler et à approfondir non seulement les sciences sociales et humaines mais aussi et surtout le débat démocratique à l'intérieur des sociétés occidentales toujours plus multiculturelles dans un monde plus global et plus polycentrique. Si la critique que fait Jean-François Bayart des Postcolonial Studies est souvent stimulante, son mépris, visible dès son titre ("carnaval académique») est totalement 
inacceptable et contraire au devoir universitaire qu'il revendique tant dans ce texte et prétend ne pas trouver dans de nombreuses études postcoloniales anglophones.

Selon lui, la France serait actuellement gagnée par une hystérie postcoloniale suspecte. En effet, depuis environ dix ans, et plus particulièrement dans les cinq dernières années, de très nombreuses publications dans la lignée postcoloniale sont apparues en France sous diverses formes qu'il s'agisse de traductions d'œuvres anglophones (Homi Bhaba, Les Lieux de la culture, 2007), d'anthologies critiques (Neil Lazarus, Penser le postcolonial, 2006), de publications de textes de colloque (M-C. Smouts, La Situation postcoloniale, 2007) ou bien encore des numéros spéciaux de revues académiques, ou généralistes dont: Les Débats, 2005 ; Mouvements, 2006 ; Esprit, 2006 ; Multitudes, 2006 ; Labyrinthe, 2006 ; Hérodote, 2006 ; Contretemps, 2006. Selon le politologue, cet engouement qui ne serait qu'une autre « mode » se ferait par « masochisme hexagonal » ou américanophilie (37).

Bayart récuse, à mon sens justement, les accusations, encore trop courantes aux USA, qui voudraient que si les Postcolonial Studies ont mis si longtemps pour être acceptées en France, c'est parce que la «France » est raciste ou indifférente ou simplement anti-américaine (31-32). Après d'autres (cf. Moura; Smouts), il montre qu'il faut comprendre cette résistance dans son contexte propre, en particulier institutionnel comme le compartimentage rigide des disciplines universitaires ou même la faillite des universités - africaines et françaises -, l'affaissement des maisons d'éditions africaines, etc (34-35). Bayart doit pourtant reconnaitre ensuite, en citant un de ses collaborateurs occasionnels (Mbembe), que « le paradoxe est qu'à cause de son insularité culturelle et du narcissisme de ses élites la France se soit coupée de ces nouveaux voyages de la pensée mondiale » (34).

Cependant, selon Bayart, de nombreux sociologues, anthropologues, politologues et historiens français auraient commencé dès les années 50 à critiquer le colonialisme et l'européocentrisme. Les noms que Bayart cite sont, comme il le fait remarquer après beaucoup d'autres, ceux qui seront d'ailleurs souvent cités par les Postcolonial Studies mêmes, tels Sartre, Fanon, Memmi, Césaire, Glissant, Balandier, et plus tard, les Mudimbe, Chamoiseau, ... (Remarquons qu'il ne cite pas une seule femme! Elles ne manquent pourtant pas! Serait-ce encore un symptôme de ce manque de sensibilité " française » ?). Que la France ait eu des critiques de la colonisation dès les années 50 et même avant d'ailleurs (cf. Lacouture, 1993), cela ne fait aucun doute. Mais ce ne sont pas les quelques noms que Bayart énumère qui font un mouvement (voir son chapitre « on a déjà donné », 20-39). Ces critiques «français » ayant précédé la vague des Postcolonial Studies ont certainement été d'autant plus courageux qu'ils ont en fait été bien peu nombreux proportionnellement. Le cas de l'Algérie est là pour le montrer : combien d'intellectuels ont osé protester et moins encore "porter les valises »? Pour un Sartre et quelques « sartristes ", combien d'intellectuels non-engagés et combien de pré-proto-LePen[istes] !

De plus, et surtout, la majorité de ceux que Bayart cite ne sont pas de la métropole mais justement des marges francophones (Caraïbes, Afrique, etc,) ou des marges françaises (des minorités, entre autres la minorité juive, qui avait été abandonnée ou trahie par une partie de la France [le gouvernement de Vichy et de nombreux Français], comme Aron, Hassner, Weil Il cite aussi Hannah Arendt plusieurs fois [33, 40, 57] comme à l'appui de sa thèse «française» alors que Arendt n'est pourtant pas française que je sache!]. Ce dernier fait 
" ethnique », Bayart ne le remarque même pas, dans une attitude typiquement française, volontairement aveugle à ce que les anti-postcolonialistes appellent très vite «communautarisme ». D'autres auteurs cités par lui sont tout récents ou très peu connus sauf dans les cercles limités, universitaires par exemple. Le cas de Franz Fanon, cité par Bayart plusieurs fois, est justement un cas «postcolonial » intéressant. Etait-il français ? Oui bien sûr, sur papier (il était martiniquais donc français), mais comme son œuvre le montre, en réalité, c'était une tout autre histoire. Et comme l'a bien montré David Macey (15), dès sa mort, il est oublié en France alors que les Postcolonial Studies l'ont très tôt « ressuscité »! Autre cas intéressant, La Renaissance orientale. Cité quatre fois par Bayart $(22,23,33,39)$, ce texte sorti en 1950 qui avance certaines thèses plus tard défendues par Edward Saïd, l'un des pères fondateurs des Postcolonial Studies, n'a jamais été republié en France. Par contre, il l'a bien été aux USA en 1976 en français (!), puis en anglais en 1984 par Columbia University Press, en pleine «mode» des Postcolonial Studies! De même, Bayart est contredit par d'autres spécialistes lorsqu'il affirme que "l'histoire connectée » qui a débattu de certaines problématiques similaires à celles des Postcolonial Studies dès les années 50, aurait rencontré en France un succès remarquable (28). Ainsi, en 2007, dans un numéro spécial sur « Histoire et mondialisation » de la Revue d'histoire moderne et contemporaine (n. 54-4bis), Bernard Thomann affirme tout le contraire (1-11).

Bayart a sans doute au moins partiellement raison d'expliquer le succès des Postcolonial Studies aux USA par des facteurs tels que les sentiments anti-français, la compétition avec l'Europe, le marketing, le carriérisme (37-38), la « cooptation en son sein des trublions les plus brillants de l'élite indigène » sur le marché américain (67). Mais il serait facile de démontrer que ces facteurs sont le lot de tous les domaines de recherche à plus ou moins divers degrés (dixit Bourdieu que Bayart cite de nombreuses fois) et pas seulement des Postcolonial Studies. De même, reprendre la critique selon laquelle les intellectuels postcoloniaux seraient en fait des "acheteurs-achetés » ("compradores », 8) me semble être de mauvaise foi. D'abord, Bayart se trompe puisque cette critique est de Appiah et non de Dirlik pour être ensuite critiquée par Dirlik (cf. Lazarus 65). Ensuite, ces intellectuels sont nécessairement de quelque part, ce qui relativise effectivement leur savoir, mais pas plus que celui, aussi gigantesque soit-il, de Bayart, sans l'accuser d'être au service de lobbies européocentristes. Enfin, même si ce livre est aussi polémique, faut-il aller jusqu'à utiliser des arguments ad hominen comme lorsqu'il traite Spivak de « demoiselle d'honneur comparée à Sartre » (21).

Les Postcolonial Studies sont un mouvement de recherche et non un dogme, et donc il y a de tout: quelques acquis, beaucoup de pistes de recherche, des idées qui se bousculent, mais aussi des tentatives de manipulations et de récupérations idéologiques douteuses, des approximations, et même des erreurs. Et que la France ait produit une quantité incroyable de thèses, articles et livres dans les 20-30 dernières années, c'est sans nul doute vrai. Cependant, selon moi, là n'est pas l'essentiel des Postcolonial Studies anglo-saxonnes et maintenant françaises et des études coloniales et postcoloniales françaises.

Pour moi, comme pour beaucoup, l’important est le débat public que les Postcolonial Studies ont réussi à provoquer depuis 1980 dans les pays anglophones et depuis 2000 en France, et que Bayart voudrait arrêter (6). Ce qu'il manque ou ce qui a manqué jusqu'à tout récemment en France, pour de multiples et complexes raisons, c'est un "débat guidé », 
d'abord dans les universités, ensuite dans les médias. Ces débats seraient "guidés » non par des journalistes ou des politiciens qui sont parfois plus ou moins informés et souvent, de par leur métier et/ou leur personnalité, trop directement intéressés, mais justement par des intellectuels engagés et spécialistes à l'image des Said, Spivak, Bhabba, et même des Bayart. Or, ce débat est absent ou remplacé par des débats superficiels ou intéressés comme le récent « débat » sur l'identité française. De manière très intéressante, les trois exemples cités par Bayart qui sortent des milieux universitaires pour amorcer ce genre de débats (littérature, musique et cinéma) sont révélateurs de l'ignorance de ce problème.

Commençons par celui qui aurait l'impact le plus vaste, le cinéma. Bayart cite un exemple (UN_ça en est même risible ! p. 33), celui de Kechiche. Un cinéaste remarquable mais dont les films, d'ailleurs tous récents $(2000,2003,2007)$ n'ont eu qu'une audience très limitée vu leur facture non et anti-hollywoodienne mais aussi justement à cause du manque de débat qui inciterait plus de gens à aller les voir. À l'inverse pour la musique, il donne de nombreux exemples (33) et il est vrai que la «France» a été une terre accueil pour cette «World music», qui est très populaire (et donc aussi très rentable). Mais on ne peut s'empêcher de penser que cela reste très ambigu puisque la musique contribue justement tout autant à renforcer les stéréotypes qui font que les Africains, les Arabes, et les autres « indigènes » sont bons pour la musique et la danse comme pour le sport d'ailleurs (On se rappelle la France Bleu-Blanc-Beur euphoriquement multiculturelle pendant quelques mois grâce à Zidane et Henry en 1998 !). Mais ces indigènes sont-ils « bons » pour les débats sur les thèmes des Postcolonial Studies (colonialisme, racisme, etc.)?

Bayart donne ensuite l'exemple de la littérature, où il est vrai que depuis longtemps des écrivains " postcoloniaux » ont pu s'exprimer sur ces thèmes. Pourtant là aussi le bilan est mitigé. Car Bayart oublie ou ignore certains faits. Ainsi Kourouma, l'un des écrivains qu'il cite comme exemple d'écrivain bien accueilli (29), a d'abord été rejeté par les éditeurs français de la métropole et a publié au Québec pour ne l'être à Paris qu'une fois qu'il eut rencontré un certain succès. De même, les prix littéraires donnés aux "indigènes de la République » auxquels il fait référence sont tout récents (après 2000) comme il le dit luimême (29), c'est-à-dire au début de la vague postcoloniale en France (Heureux hasard!). Soyons sérieux : le premier "non-blanc » à l'Académie française est Senghor (un écrivain et penseur remarquable mais au discours aussi très francophoniste, conciliant) en 1984, et puis le deuxième, François Cheng, en 2002, et la troisième, Assia Djebar, en 2005!!! Bayart nous dit aussi que de nombreux critiques des Postcolonial Studies auraient été souvent invités en France (32) mais sans pourtant nous donner aucune référence. Il serait facile de montrer que «souvent» est relatif, qu'«en France» veut dire dans les universités parisiennes, et que cet accueil des spécialistes des Postcolonial Studies n’est significatif que depuis l'an 2000.

Autre preuve que Bayart ne voit pas ces Postcolonial Studies dans le rôle qu'elles peuvent jouer dans l'espace public, c'est qu'il ne fait aucune mention de la télévision. Nulle mention du fait que jusqu'en l'an 2006, il n'y avait aucun présentateur de couleur sur les écrans français (voir l'article intitulé «Une télévision française toujours trop pâle » paru dans Le Monde du 12 novembre 2008)! Nulle mention que, sauf pour deux ou trois exceptions (d'ailleurs lavées très blanc), jusqu'à tout récemment, il n'y avait aucun sitcom français qui incluait des minorités ethniques. Car, s'il est vrai, comme Bayart l'écrit, que certains dans les Postcolonial Studies ont trop souvent péché par un excès d'attention aux 
discours et pas assez aux " réalités », d'autres venant aussi des Postcolonial Studies, comme Said, During, Parry et d'autres (Lazarus 150), avaient déjà dénoncé ce problème. Le fait est que ces Postcolonial Studies sont aussi très sensibles aux réalités contemporaines entre autres racistes comme le délit de faciès dont certains font les frais tous les jours dans leurs recherches d'emploi ou de logement ou dans leur travail même (voir l'article « Moi, Mustapha Kessous ...» paru dans Le Monde du 23 septembre 2009), et à l'absence de représentations multiculturelles dans les espaces publics, fait essentiel pour promouvoir un multiculturalisme qui alors contribuerait à dissoudre les tensions dans les banlieues, et entre banlieues et «métropole », et à dissoudre les idées d'un certain nombre de convaincus qui votent FN. Il est alors trop facile d'accuser Sarkozy d'avoir fait une erreur monumentale à Dakar (25). En fait, cette erreur est révélatrice de l'absence de débat en France et n'est le résultat que de l'indifférence, l'ignorance ou le déni des problèmes racistes et ethniques au moins en partie dus aux colonialismes et plus encore au non-débat sérieux de la part des intellectuels et artistes français.

Pour conclure, le défaut majeur de ce livre à côté de ses nombreuses qualités c'est le mépris pour les Postcolonial Studies et pour le débat public qu'elles ont provoqué aux USA. Un livre À LIRE donc, mais de manière très critique pour pouvoir justement continuer le débat commencé il y a trop peu de temps en France! 


\section{Bibliographie}

Bayart, J-F. Les Etudes Postcoloniales: Un carnaval académique. Paris : Karthala, 2010.

Girard, L. «Une télévision française toujours trop pâle », Le Monde, 12 novembre 2008, accès en ligne le 12 novembre 2008.

Kessous, M. "Moi, Mustapha Kessous, journaliste au 'Monde' et victime du racisme », Le Monde, 24 septembre 2009, accès en ligne le 23 septembre 2009.

Lacouture, J. Le Désempire : Figures et thèmes de l'anticolonisme, Paris : Denoël, 1993.

Lazarus, N. (Dir.). Penser le Postcolonial: Une introduction critique. (traduit de l'anglais par M. Groulez, C. Jaquet et H. Quiniou). Paris: Amsterdam, 2006.

Macey, D. Fran₹ Fanon: A Biography. New York : Picador, 2001.

Smouts, M-C. La Situation postcoloniale. Paris : Presses de Sciences Politiques, 2007. 